\title{
An Improved Critical-Edge Model for Finding Optimal Contraflow Links Considering the Influence of Intersections
}

\author{
Junwen Mo, ${ }^{1}$ Mingxia Gao $\left(\mathbb{D},{ }^{2}\right.$ and Liqiao Liu $^{2}$ \\ ${ }^{1}$ School of Civil Engineering of Lanzhou Jiaotong University, Lanzhou, Gansu 730070, China \\ ${ }^{2}$ School of Traffic and Transportation of Lanzhou Jiaotong University, Lanzhou, Gansu 730070, China \\ Correspondence should be addressed to Mingxia Gao; mxgao@mail.lzjtu.cn
}

Received 15 March 2019; Revised 4 June 2019; Accepted 2 July 2019; Published 10 July 2019

Academic Editor: Alexander Paz

Copyright (c) 2019 Junwen Mo et al. This is an open access article distributed under the Creative Commons Attribution License, which permits unrestricted use, distribution, and reproduction in any medium, provided the original work is properly cited.

\begin{abstract}
A reversible roadway (contraflow) is one in which the direction of traffic flow in one or more lanes is reversed to the opposing direction for some period of time. Reversible roadways are most commonly used for accommodating directionally imbalanced traffic associated with daily commuter periods. Reversible lanes also have been widely used, in recent years, for evacuating major metropolitan regions threatened by hurricanes and other disasters. One important problem in the practice of evacuation traffic organization is the choice of road links for contraflow. Most research on the choice of contraflow links does not consider the influence of intersections, which leads to overestimation of evacuation capacity especially in congested urban road networks. We abstract an evacuation road network as a special network with directional node-weights by considering the capacity of intersection movements as directed weights of nodes. We define the critical edge for increasing the maximum flow value of such network as the one that can maximize the range of flow value increase by expanding its capacity. We obtain alternative links for contraflow by searching critical edges in such network. We presented a modified algorithm for finding such critical edges on the basis of the maximal capacity path algorithm for the classical maximum flow problem. We also provided a numerical example and tested the effects through traffic simulation. Our results show that the results considering the influence of intersections are more reasonable than those ignoring it and that taking the intersection effects into account enables us to reduce the total evacuation time.
\end{abstract}

\section{Introduction}

A large area evacuation is usually needed for emergency management in the event of natural or man-made disasters, which produce a dramatic, unbalanced traffic surge. These circumstances require special measures for traffic management and control to avoid traffic jam and delays on some roads and intersections.

Reversible roadways are most commonly used for traffic management associated with daily commuter periods [1]. They also have been used regularly to increase outbound evacuation route capacity during emergencies such as hurricanes. Lane reversals were first applied to the evacuation of Hurricane Georges and Hurricane Floyd in the United States in the 1990s and subsequently used in the evacuation in Louisiana and Mississippi [2].

Previous studies of lane reversal design fall into two types. The first focuses on identifying optimal positions (also called additional intersections) as the starting points to change lane directions in a section of highway. This approach is mainly applied to hurricane related evacuations. Wolshon discussed some operational practices for lane reversals during hurricane evacuation and laid special stress on the problem of vehicles rerouting at the entrance and exit of reversed lanes $[3,4]$. Theodoulou and Wolshon analyzed the effects of the contraflow plan used in hurricane evacuation in New Orleans using the CORSIM simulation software [5, 6]. Zhou Yafei et al. assessed different methods and plans for determining the starting points of reversed lanes in highway networks using the Cell Transmission Model (CTM) [7].

The second type of lane reversal research examines traffic evacuation relying upon urban roads and highways with high density, in which the key consideration is selecting appropriate links for contraflow. Shekhar and Kim proved that selecting optimal reversal links in a road network is an NPhard problem and introduced the overload degree parameter. 
They then designed a greedy heuristic to handle scenarios with a high overload degree and a bottleneck relief heuristic for situations with low overload degree [8]. Wang and Ip et al. studied the development of a new approach for resource planning using contraflow techniques, in which contraflow and road repairs are simultaneously considered [9]. Wang et al. presented a multiple-objective optimization model for selecting reversal links and a two-layer algorithm to solve this model considering both evacuation priorities and the setup time for the contraflow operation [10]. Min and Lee defined the maximum throughput flow and proposed a contraflow evacuation routing algorithm using reverse shortest paths and maximum throughput flows [11]. Hadas et al. developed a model for designing an optimal network minimizing both evacuation time and network constructions costs [12]. Gao et al. studied the problem of selecting contraflow links in traffic evacuation with a network optimization technique $[13,14]$.

In most studies of link-selection for contraflow, the influence of intersections has received insufficient attention. It is common for road sections to be managed with reversal lanes while intersections operate as usual. However, the effectiveness of increased capacity from contraflow depends greatly on the capacity of intersections. This is particularly evident in crowded urban road networks. Ignoring the effects of intersection in the selection of reversal links in a road network may result in overestimating expected capacity.

In this paper, we propose an improved critical edge model of the maximum flow to optimize the link selection for contraflow. We abstract the evacuation road network as a special network by expressing the capacity of turning lanes at intersection entrance as directional node weights. We define the critical edge of the maximum flow increase as the edge that brings the largest increase to the maximum flow value of the network once its capacity is expanded. We obtain alternative links for contraflow by searching critical and subcritical edges for the maximum flow increase. We present a modified algorithm for finding such critical edges on the basis of the maximal capacity path algorithm for the classical maximum flow problem. We also provided a numerical example and tested the effects through traffic simulation.

This paper is organized as follows. Section 2 illustrates the formulation of the model and its characteristic. Section 3 presents a solution algorithm. Section 4 provides a numerical example to demonstrate the application. Section 5 presents our conclusions.

\section{Model Formulation}

2.1. Modeling Ideas. In traditional network flow theory, the critical edge of the maximum flow refers to the side that provides the maximum loss of the maximum flow value when it is interrupted. This measure is often used to analyze the effect of a failure of a road section or a sudden interruption of a line on a communication network [15]. As mentioned in the introduction, we abstract the evacuation network as a special network with the directional node weights and define the critical edge of the maximum flow increase as the edge that brings the largest increase to the maximum flow value once capacity is expanded.

To improve the capacity of evacuation route, it is usually necessary to find one or more optimal links for reversing lanes (i.e., contraflow). The core of this optimization is the selection of road links, with the links considered playing an important role in improving the overall evacuation capacity of the road network. Although the traffic flow distribution is not necessarily the maximum flow, the road link corresponding to the critical edge of the maximum flow increase plays a key role. By abstracting the evacuation network as a special network with directional node weights and searching for the critical edge of maximum flow increase in it, the alternative road links suitable for contraflow can be obtained when the influence of intersection is taken into account.

2.2. Symbol and Variable Definition. Based on the above considerations, we consider the optimization of the reversal links selection as the critical edge problem of the maximum flow increase in the network with directional node weights. The following material presents the symbols and variables we use in our approach.

Treating road links as arcs and intersections as nodes, we express the evacuation road network as a directional network $\boldsymbol{G}=(\boldsymbol{V}, \boldsymbol{A}, \boldsymbol{C}, \boldsymbol{U}) \cdot \boldsymbol{V}=\{i / i=1,2, \cdots, n\}$ is the set of nodes (intersections) with $|\boldsymbol{V}|=n$, and $\boldsymbol{A}=\{(i, j) / i, j=1,2 \cdots, n\}$ is the set of $\operatorname{arcs}\left(\right.$ links) with $|\boldsymbol{A}|=m . C=\left\{c_{i j} / i, j=1,2, \cdots n\right\}$ is the set of road capacities with $c_{i j}$ indicating the capacity of link $(i, j) . U=\left\{u_{i j k} / i, j, k=1,2, \cdots n\right\}$ is the set of turn capacities with $u_{i j k}$ indicating the capacity of the turn direction $i-j-k$ at node $j$. For any edge $(i, j)$ between an $\mathrm{O}-$ $\mathrm{D}$ pair $(s, t)$ in the network $\boldsymbol{G}$, capacity increase is expressed as $c_{i j}=\lambda c_{i j}, \lambda>1$. For this increase, we express the new maximum flow value as $v_{\mathbf{G}+(i, j)}$. If there exists an edge $\left(i^{*}, j^{*}\right)$ and the formula $v_{\mathbf{G +}\left(i^{*}, j^{*}\right)} \geq v_{\mathbf{G +}(i, j)}$ is true for all edge $(i, j)$ in the network $G$, then the edge $\left(i^{*}, j^{*}\right)$ is called the critical edge of maximum flow increase for the network $\boldsymbol{G}$. The critical edge and the subcritical edge of the maximum flow increase can be found by calculating the maximum flow after capacity expansion for all of the edges and sorting in descending order according to the maximum flow value. The selected edges can be regarded as the alternative links of lane reversal setting. One or more links can be selected to expand the capacity through contraflow according to the real evacuation demand, with the expectation that the overall evacuation capacity of the road network will be improved.

\section{Algorithm Design}

3.1. General Framework of Algorithm. According to its definition, the critical edge of the maximum flow increase can be found by calculating the maximum flow of the new network after the expansion of each edge and comparing the amplitude of the increase for all edges. There are many unnecessary calculations in this natural algorithm because not all edge expansions contribute to the maximum flow. According to the flow decomposition theorem, the maximum flow value can be improved only if the edge located on the specific path is expanded. 
Therefore, we make use of the following ideas. First, we use the modified maximum capacity path algorithm to calculate the maximum flow of the initial network and record the augmented path found in each step for the augmenting flow value. Then we determine whether each edge of the network belongs to one of the augmented paths. If the edge is not included in any augmented path, its expansion has no effect on the maximum flow. Otherwise, the maximum flow of the new network can be calculated after the edge is expanded. Finally, we find the critical edge of the maximum flow increase by sorting the increment brought by capacity expansion in descending order. To improve the efficiency, we use the maximum flow of the original network as the initial feasible flow when calculating the maximum flow of a new network after an edge expansion. The detailed calculation steps are as follows.

Step 1. Let $\boldsymbol{R}=\boldsymbol{A}$ (the set of links) $\operatorname{arc}=\phi$, and calculate the maximum flow of overall network $\boldsymbol{G}$ using the modified maximum capacity path algorithm, recording the maximum flow as $f_{G}$, the flow value as $v_{G}$, and the augmented paths as $L_{1}, L_{2}, L_{3}, \ldots, L$.

Step 2. If $\boldsymbol{R}=\phi$, go to Step 5. Otherwise, taking any $(i, j) \in \boldsymbol{R}$, find an augmented path including $(i, j)$; if $(i, j)$ is not included in any augmented path, go to Step 4. Otherwise, go to Step 3.

Step 3. Taking the maximum flow $\boldsymbol{f}_{\mathrm{G}}$ as the initial feasible flow of network $\boldsymbol{G}_{+(i, j)}$ (the new network after expanding edge $(i, j))$, find the maximum flow of the new network $\boldsymbol{G}_{+(i, j)}$, recording the flow value as $v_{G+(i, j)}$. Let $\boldsymbol{R}=\boldsymbol{R}-\{(i, j)\}$ and go to Step 2.

Step 4. The expansion of edge $(i, j)$ has no effect on the maximum flow of network $\boldsymbol{G}$, so let $\boldsymbol{R}=\boldsymbol{R}-\{(i, j)\}$ and go to Step 2.

Step 5. Compare the maximum flow value of the new network after expansion of each edge and sort in descending order.

3.2. Algorithm for Maximum Flow of the Network with Directional Node Weights. The algorithm needs to search for the maximum flow repeatedly in the network with directional node weights. However, the traditional maximum flow problem is based on an ordinary network and lacks support for the directional node weights. Therefore, we must find the maximum flow in the special network with directional node weights. We proposed a modified algorithm on the basis of the maximum capacity path algorithm of the classical maximum flow problem with some improvements for critical steps, including the incremental network construction, the maximum capacity path search, and the flow increase along the augmented path.

For convenience, let $\boldsymbol{P}$ represent the augmented path with $\boldsymbol{P}^{+}$and $\boldsymbol{P}^{-}$representing the sets of forward and backward arcs in the augmented path, respectively. Let node 1 and node $n$ represent the starting and ending point, respectively. The specific steps of the algorithm are then as follows.
Step 1. Give an initial feasible flow $\boldsymbol{f}$ to network $\boldsymbol{G}$, where $\boldsymbol{f}=$ $\left\{f_{i j}, f_{i j k} /(i, j) \in \boldsymbol{A},(j, k) \in \boldsymbol{A}\right\}$.

Step 2. Construct an incremental network expressed as $G(f)=(V, A(f), C(f), U(f))$ for flow $f$, where $A(f)=$ $A^{+}(f) \cup A^{-}(f)$ is the set of arcs in which $A^{+}(f)=$ $\left\{(i, j) /(i, j) \in \boldsymbol{A}, f_{i j}<c_{i j}\right\}$, and $\boldsymbol{A}^{-}(\boldsymbol{f})=\left\{(i, j) /(j, i) \in \boldsymbol{A}, f_{j i}>\right.$ $0\} . \boldsymbol{C}(\boldsymbol{f})$ and $\boldsymbol{U}(\boldsymbol{f})$ represent the capacity of arcs and turningdirections, respectively, and are determined by the following formulas:

$$
\begin{aligned}
& c_{i j}(\boldsymbol{f})= \begin{cases}c_{i j}-f_{i j} & (i, j) \in \boldsymbol{A}^{+}(\boldsymbol{f}) \\
f_{j i} & (i, j) \in \boldsymbol{A}^{-}(\boldsymbol{f})\end{cases} \\
& u_{i j k}(\boldsymbol{f}) \\
& = \begin{cases}u_{i j k}-f_{i j k} & (i, j) \in \boldsymbol{A}^{+}(\boldsymbol{f}) \text { and }(j, k) \in \boldsymbol{A}^{+}(\boldsymbol{f}) \\
f_{k j i} & (i, j) \in \boldsymbol{A}^{-}(\boldsymbol{f}) \text { and }(j, k) \in \boldsymbol{A}^{-}(\boldsymbol{f}) .\end{cases}
\end{aligned}
$$

Step 3. Find the path $\boldsymbol{p}$ with the largest capacity from source node 1 to end node $n$ in the incremental network and note its capacity as $\theta$. If there is no such route, the algorithm ends, indicating that the maximum flow has been found. Otherwise go to Step 4

Step 4. Increase the flow by value $\theta$ for the network $\boldsymbol{G}$ along path $\boldsymbol{p}$ using the following formulas:

$$
\begin{aligned}
f_{i j} & = \begin{cases}f_{i j}+\theta & (i, j) \in \boldsymbol{P}^{+} \\
f_{i j}-\theta & (j, i) \in \boldsymbol{P}^{-} \\
f_{i j} & \text { else }\end{cases} \\
f_{i j k} & = \begin{cases}f_{i j k}+\theta & (i, j) \in \boldsymbol{P}^{+} \text {and }(j, k) \in \boldsymbol{P}^{+} \\
f_{i j k}-\theta & (j, i) \in \boldsymbol{P}^{-} \\
f_{i j k} & \text { else }(k, j) \in \boldsymbol{P}^{-}\end{cases}
\end{aligned}
$$

Step 5. Calculate and record the current flow value as $f^{*}=$ $\sum_{i}\left(f_{1 i}-f_{i 1}\right)$. Go to Step 2 .

The search for the maximum capacity path in Step 2 is solved by the labeling correction algorithm that labels arcs instead of nodes. The details of this algorithm can be found in our previous work [16].

\section{Example}

4.1. Initial Data. We analyzed our proposed method by taking the abstract road network shown in Figure 1 as an example. In Figure 1, nodes 1, 2, and 3 represent the evacuation source, and node 17 represents the evacuation end. The evacuation demand generated by nodes 1,2 , and 3 during evacuation needs to be delivered to node 17 in its entirety. For simplicity, we assume that the capacity and other information of all links are two-way symmetric. Table 1 shows the basic road link information required for calculation and simulation, and Table 2 shows the turning capacity of each intersection. 
TABLE 1: The basic information of road links.

\begin{tabular}{|c|c|c|c|c|c|c|}
\hline $\begin{array}{l}\text { Directional } \\
\text { links }\end{array}$ & $\begin{array}{c}\text { Free-flow speed } \\
v_{f} /(\mathrm{km} / \mathrm{h})\end{array}$ & $\begin{array}{c}\text { Minimum speed } \\
v_{\min } /(\mathrm{km} / \mathrm{h})\end{array}$ & $\begin{array}{l}\text { Length } \\
L / m\end{array}$ & $\begin{array}{c}\text { The number of } \\
\text { lanes } \\
\text { Lan }\end{array}$ & $\begin{array}{l}\text { Capacity } \\
c_{i j} /(p c u / h)\end{array}$ & $\begin{array}{c}\text { Jam density } \\
k_{j} /(p c u / k m / l a n)\end{array}$ \\
\hline$(1,5) /(5,1)$ & 35 & 15 & 600 & 2 & 1900 & 140 \\
\hline$(1,6) /(6,1)$ & 35 & 10 & 600 & 1 & 1100 & 140 \\
\hline$(2,12) /(12,2)$ & 30 & 5 & 600 & 1 & 1200 & 140 \\
\hline$(2,13) /(13,2)$ & 30 & 5 & 600 & 2 & 2100 & 140 \\
\hline$(3,7) /(7,3)$ & 35 & 12 & 600 & 2 & 1850 & 140 \\
\hline$(3,8) /(8,3)$ & 35 & 10 & 600 & 1 & 1200 & 140 \\
\hline$(8,7)(7,8)$ & 40 & 15 & 800 & 2 & 2100 & 130 \\
\hline$(7,6) /(6,7)$ & 40 & 10 & 900 & 2 & 1900 & 130 \\
\hline$(6,5) /(5,6)$ & 42 & 13 & 900 & 2 & 1850 & 135 \\
\hline$(5,4) /(4,5)$ & 50 & 15 & 1130 & 2 & 2000 & 150 \\
\hline$(8,13) /(13,8)$ & 48 & 16 & 1150 & 3 & 3050 & 153 \\
\hline$(7,12) /(12,7)$ & 50 & 15 & 1050 & 2 & 1700 & 160 \\
\hline$(6,11) /(11,6)$ & 46 & 14 & 1000 & 1 & 1100 & 150 \\
\hline$(5,10) /(10,5)$ & 45 & 16 & 1080 & 2 & 2200 & 145 \\
\hline$(4,9) /(9,4)$ & 55 & 15 & 1500 & 1 & 1300 & 155 \\
\hline$(13,12) /(12,13)$ & 38 & 12 & 860 & 2 & 1850 & 150 \\
\hline$(12,11) /(11,12)$ & 40 & 15 & 750 & 2 & 1950 & 140 \\
\hline$(11,10) /(10,11)$ & 36 & 12 & 800 & 2 & 2100 & 145 \\
\hline$(10,9) /(9,10)$ & 43 & 15 & 1000 & 1 & 1200 & 155 \\
\hline$(13,15) /(15,13)$ & 45 & 15 & 1160 & 3 & 2500 & 140 \\
\hline$(11,14) /(14,11)$ & 36 & 10 & 900 & 2 & 1800 & 150 \\
\hline$(10,14) /(14,10)$ & 46 & 15 & 1200 & 1 & 1050 & 135 \\
\hline$(9,17) /(17,9)$ & 50 & 16 & 1600 & 2 & 1970 & 145 \\
\hline$(14,16)(16,14)$ & 35 & 10 & 780 & 2 & 2050 & 135 \\
\hline$(15,16) /(16,15)$ & 56 & 15 & 1450 & 3 & 2750 & 140 \\
\hline$(16,17) /(17,16)$ & 50 & 15 & 1560 & 3 & 2850 & 145 \\
\hline
\end{tabular}

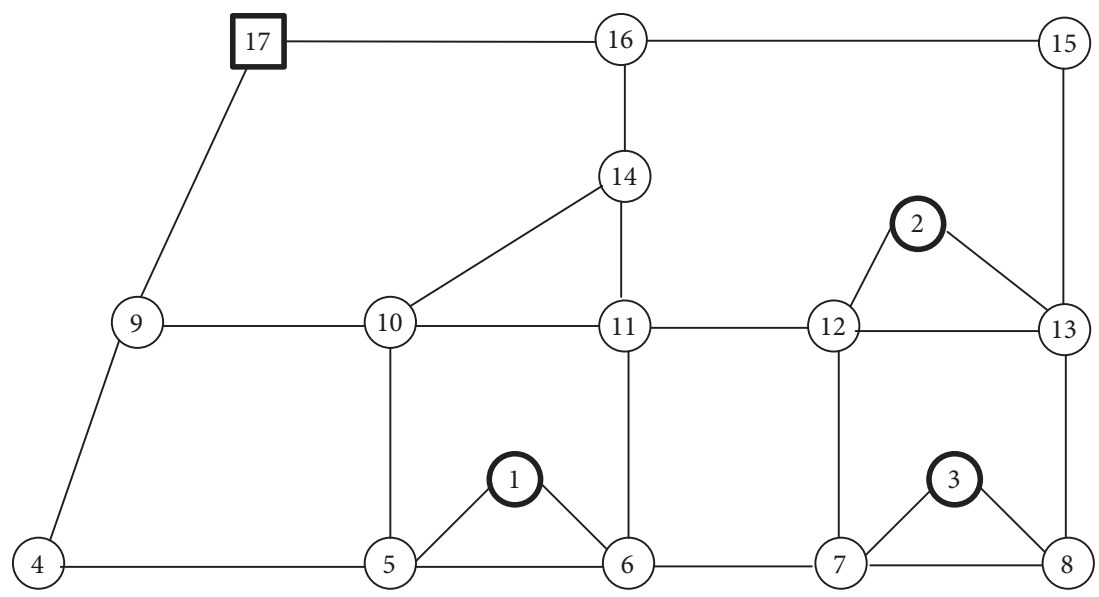

FIGURE 1: The abstract evacuation road network.

4.2. Optimization Results. Our method determined that the critical edge of the maximum flow increase of the network shown in Figure 1 is edge $(4,9)$. The maximum flow value of the network increased by 540 after expanding the capacity of link $(4,9)$ by implementing contraflow on (i.e., reversing) link $(9,4)$. In addition, using our previous method without considering the influence of the intersections [14], we found that the critical edge of the maximum flow increase of the network is link $(14,16)$, with the increment of the maximum flow value being only 130 after capacity expansion. The reason 
TABLE 2: Capacity of turning directions at intersections.

\begin{tabular}{|c|c|c|}
\hline Intersections & Turning directions & $\begin{array}{c}\text { Capacity } \\
u_{i j k} /(p c u / h)\end{array}$ \\
\hline 4 & $5-4-9$ & 1800 \\
\hline \multirow{4}{*}{5} & $6-5-4$ & 960 \\
\hline & $6-5-10$ & 1060 \\
\hline & $1-5-4$ & 940 \\
\hline & $1-5-10$ & 1130 \\
\hline \multirow{4}{*}{6} & $1-6-5$ & 600 \\
\hline & $1-6-11$ & 570 \\
\hline & $7-6-5$ & 1100 \\
\hline & $7-6-11$ & 1220 \\
\hline \multirow{4}{*}{7} & $8-7-6$ & 1230 \\
\hline & $8-7-12$ & 1350 \\
\hline & $3-7-6$ & 1180 \\
\hline & $3-7-12$ & 1260 \\
\hline \multirow{2}{*}{8} & $3-8-7$ & 560 \\
\hline & $3-8-13$ & 680 \\
\hline \multirow{2}{*}{9} & $4-9-17$ & 1300 \\
\hline & $10-9-17$ & 720 \\
\hline \multirow{4}{*}{10} & $5-10-9$ & 1000 \\
\hline & $5-10-14$ & 1100 \\
\hline & $11-10-9$ & 1200 \\
\hline & $11-10-14$ & 960 \\
\hline \multirow{4}{*}{11} & $6-11-10$ & 540 \\
\hline & $6-11-14$ & 720 \\
\hline & $12-11-10$ & 1120 \\
\hline & $12-11-14$ & 1200 \\
\hline \multirow{3}{*}{12} & $7-12-11$ & 1400 \\
\hline & $13-12-11$ & 980 \\
\hline & $2-12-11$ & 540 \\
\hline \multirow{4}{*}{13} & $8-13-12$ & 1600 \\
\hline & 8-13-15 & 1350 \\
\hline & $2-13-12$ & 1050 \\
\hline & 2-13-15 & 1200 \\
\hline \multirow{2}{*}{14} & $10-14-16$ & 760 \\
\hline & $11-14-16$ & 1500 \\
\hline 15 & $13-15-16$ & 1900 \\
\hline \multirow{2}{*}{16} & 14-16-17 & 1200 \\
\hline & $15-16-17$ & 1600 \\
\hline
\end{tabular}

for this difference is that although link $(14,16)$ is most important when the influence of the intersection is not considered, the objective existence of the traffic capacity limitation of the intersection makes the capacity expansion of the link unable to achieve the expected effect. Although the traffic capacity of link $(14,16)$ is increased through reverse management, the traffic capacity of the related turning direction 14-16-17 is very low, which limits the overall rise in capacity of the network. The specific results of calculation and comparison are shown in Table 3.
4.3. Simulation Analysis. Table 3 shows that the critical links of maximum flow increase and the increase range of flow value after link expansion are both different when ignoring or considering the intersection influence in the optimization process. To further analyze the impact of different expansion schemes on evacuation efficiency, we assigned given evacuation demands to the original road network and calculated the results both ignoring and considering the intersection effects. We also calculated the overall evacuation time separately for the three different scenarios. The process of evacuation traffic assignment is realized by a system optimal dynamic traffic assignment model proposed previously by the author $[17,18]$. This process needs information such as the number of vehicles to be evacuated, the length of the road link, the capacity, and the speed of the free flow as given in Table 1.

We used three demand patterns for simulation analysis, as follows:

pattern 1: $D_{1-17}=800 \mathrm{pcu}$ (Passenger Car Unit), $D_{2-17}=900$ pcu, $D_{3-17}=1000$ pcu;

pattern 2: $D_{1-17}=600 \mathrm{pcu}, D_{2-17}=800 \mathrm{pcu}, D_{3-17}=700$ pcu;

pattern 3: $D_{1-17}=300 \mathrm{pcu}, D_{2-17}=500 \mathrm{pcu}, D_{3-17}=400$ pcu.

In all three scenarios, the aim was to deliver all traffic from source nodes 1, 2, and 3 to end node 17. The variation is in the demand quantity. For example, in demand pattern 1 , the evacuation demand generated by node 1 is 800 (pcu), whereas in demand pattern 2 , the evacuation demand from node 1 is $600(\mathrm{pcu})$. We performed simulation experiments in the three demand patterns. Table 4 provides the evacuation time results.

Table 4 shows that the evacuation time can be shortened in varying degrees after link expansion under the three demand patterns, regardless of the optimization process. However, the expansion effect of the selected road link is better when considering the influence of intersection, which becomes even more obvious in the case of large evacuation demand. For example, the expansion of links $(14,16)$ and $(4,9)$ shortened the total evacuation time by 3 minutes and 5 minutes, respectively, under the lower demand pattern 3 , a small difference. However, under pattern 1 with high demand, the evacuation time was shortened by 13 minutes when considering the influence of intersection with contraflow on link $(4,9)$, while the evacuation time was only reduced by 5 minutes when ignoring the influence of intersection with contraflow on link $(14,16)$.

\section{Conclusions}

Lane reversal, also known as contraflow, is a typical measure in evacuation traffic organization. In this paper, we have studied the method for selecting road links for contraflow taking the influence of intersection into account. We abstracted the evacuation road network as a special network with directional node weights and identified alternative links for contraflow by searching for the critical edge of the maximum flow increase flow in the network. To handle the directional weights of nodes, we improved the classical maximum capacity 
TABLE 3: Optimization results for critical links of maximum flow increase of the network both ignoring and considering intersection influence.

\begin{tabular}{lcccc}
\hline $\begin{array}{l}\text { Intersection } \\
\text { influence }\end{array}$ & $\begin{array}{c}\text { Critical links for capacity } \\
\text { expansion }\end{array}$ & $\begin{array}{c}\text { Maximum flow value after } \\
\text { expansion } /(\mathrm{pcu} / \mathrm{h})\end{array}$ & $\begin{array}{c}\text { Improvement range of } \\
\text { maximum flow } /(\mathrm{pcu} / \mathrm{h})\end{array}$ & $\begin{array}{c}\text { Maximum flow value of } \\
\text { original network/(pcu/h) }\end{array}$ \\
\hline Ignored & $(14,16)$ & 2780 & 130 & 2650 \\
Considered & $(4,9)$ & 3190 & 540 & \\
\hline
\end{tabular}

TABLE 4: Simulated evacuation time after capacity expansion of critical links for different demand patterns (minute).

\begin{tabular}{lcccc}
\hline \multirow{2}{*}{ Optimal conditions } & \multicolumn{2}{c}{ critical links for expansion } & Demand mode \\
& & Pattern 1 & Pattern 2 & 68 \\
\hline Original network & none & 78 (minutes) & 49 \\
Ignoring intersection & $(14,16)$ & 73 & 63 & 46 \\
Considering intersection & $(4,9)$ & 65 & 60 & 44 \\
\hline
\end{tabular}

algorithm for this problem. We analyzed the validity of the method by a numerical example.

Our method is predicated on the premise that the traffic capacity of road links and intersections is fixed. However, capacity may be dynamically changed due to sudden interruptions or unavailability of roads during evacuation as the disaster changes (e.g., flooding). Thus, it is necessary to consider the dynamic changes of traffic capacity and study the optimal selection of dynamic lane reversal in the future.

\section{Data Availability}

The data used to support the findings of this study are available from the corresponding author upon request.

\section{Conflicts of Interest}

The authors declare that they have no conflicts of interest.

\section{Acknowledgments}

We thank LetPub (www.letpub.com) for its linguistic assistance during the preparation of this manuscript. This paper is supported by the National Natural Science Foundation Project of China (No. 71661021; 61563028) and the science plan project of Lanzhou city, China (No. 2018-4-34).

\section{References}

[1] H. J. Hoose, "Planning effective reversible lane control," Traffic Quarterly, vol. 17, no. 3, pp. 408-413, 1963.

[2] E. Urbina and B. Wolshon, "National review of hurricane evacuation plans and policies: a comparison and contrast of state practices," Transportation Research Part A: Policy and Practice, vol. 37, no. 3, pp. 257-275, 2003.

[3] B. Wolshon, "One-way-out: contraflow freeway operation for hurricane evacuation," Natural Hazards Review, vol. 2, no. 3, pp. 105-112, 2001.

[4] B. Wolshon, "Planning for the evacuation of New Orleans," ITE Journal, vol. 72, no. 2, pp. 44-49, 2002.

[5] G. Theodoulou and B. Wlshon, "Modeling and analyses of freeway contraflow to improve future evacuations," in Proceedings of the 83rd Annual Meeting of the Transportation Research
Board, Transportation Research Board, Washington, D.C., USA, January 2004.

[6] E. Lim and B. Wolshon, "Modeling and performance assessment of contraflow evacuation termination points," in Proceedings of the 84th Annual Meeting of the Transportation Research Board, vol. January 2005, pp. 118-128, Transportation Research Board, Washington, D.C., USA.

[7] Y. F. Zhou, J. Cai, and X. N. Cheng, "Contraflow operation in emergency traffic evacuation," Science \& Technology Review, vol. 31, no. 28-29, pp. 43-47, 2013 (Chinese).

[8] S. Kim, S. Shekhar, and M. Min, "Contraflow transportation network reconfiguration for evacuation route planning," IEEE Transactions on Knowledge and Data Engineering, vol. 20, no. 8, pp. 1115-1129, 2008.

[9] J. W. Wang, W. H. Ip, and W. J. Zhang, "An integrated road construction and resource planning approach to the evacuation of victims from single source to multiple destinations," IEEE Transactions on Intelligent Transportation Systems, vol. 11, no. 2, pp. 277-289, 2010.

[10] J. W. Wang, H. F. Wang, W. J. Zhang, W. H. Ip, and K. Furuta, "Evacuation planning based on the contraflow technique with consideration of evacuation priorities and traffic setup time," IEEE Transactions on Intelligent Transportation Systems, vol. 14, no. 1, pp. 480-485, 2013.

[11] M. Min and J. Lee, "Maximum throughput flow-based contraflow evacuation routing algorithm," in Proceedings of the IEEE International Conference on Pervasive Computing and Communications Workshops (PerCom Workshops '13), pp. 511516, IEEE, San Diego, Claif, USA, March 2013.

[12] Y. Hadas and A. Laor, "Network design model with evacuation constraints," Transportation Research Part A: Policy and Practice, vol. 47, pp. 1-9, 2013.

[13] G. Mingxia and W. Xuemei, "Study on contra flow management based on the theory of minimum cut," China Sciencepaper, vol. 10, no. 19, pp. 2295-2297, 2015 (Chinese).

[14] M.-X. Gao, W.-Y. Hong, and Q.-Q. Si, “Optimization of contraflow links in traffic evacuation based on the critical edge for improving maximum flow of a network," Journal of Transportation Systems Engineering and Information Technology, vol. 16, no. 3, pp. 194-199, 2016 (Chinese).

[15] C. F. Shi and Y. F. Xu, "Vital-edge with the maximum flow in the traffic network," System Engineering, vol. 27, no. 9, pp. 5559, 2009 (Chinese).

[16] M.-X. Gao and G.-G. He, "An Arc labeling algorithm for shortest path problem considering turn penalties and prohibitions at 
intersections," Journal of Lanzhou Jiaotong University, vol. 30, no. 6, pp. 111-114, 2011.

[17] M. X. Gao and G. G. He, "A system optimal route and departure time choice model for evacuation planning," System Engineering, vol. 27, no. 6, pp. 73-77, 2009 (Chinese).

[18] M. X. Gao, "A node model capturing turning lane capacity and physical queuing for the dynamic network loading problem," Mathematical Problems in Engineering, vol. 2012, Article ID 542459, 14 pages, 2012. 


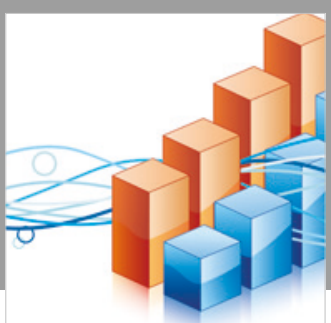

Advances in

Operations Research

\section{-n-m}
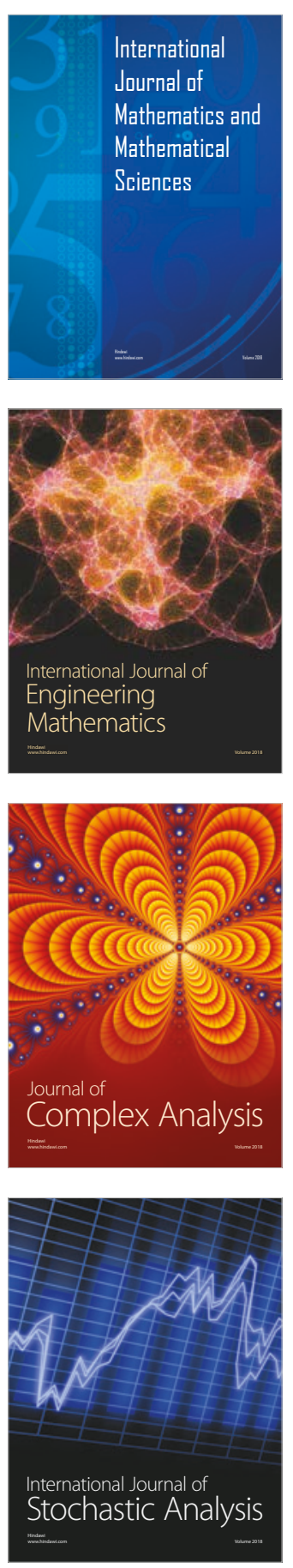
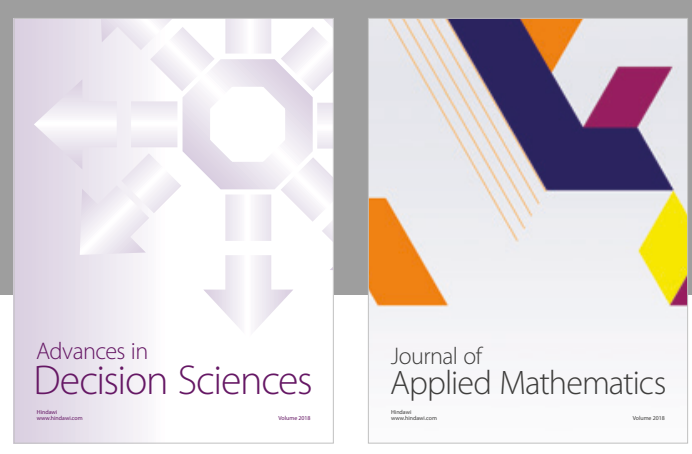

Journal of

Applied Mathematics
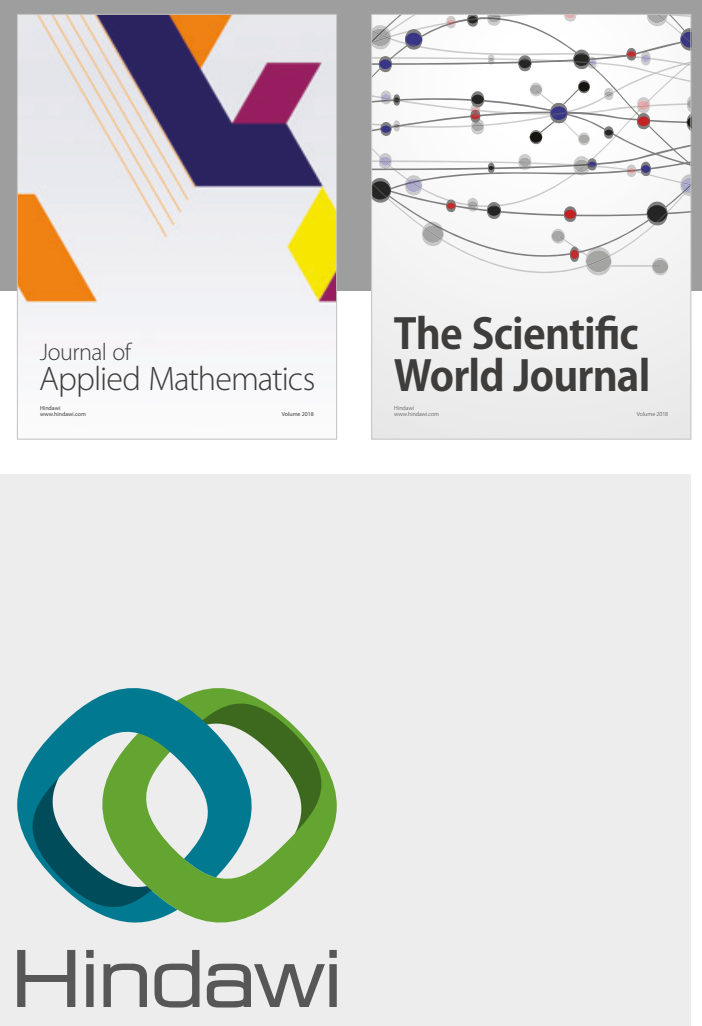

Submit your manuscripts at

www.hindawi.com

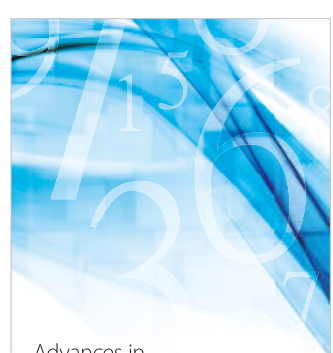

Advances in
Numerical Analysis
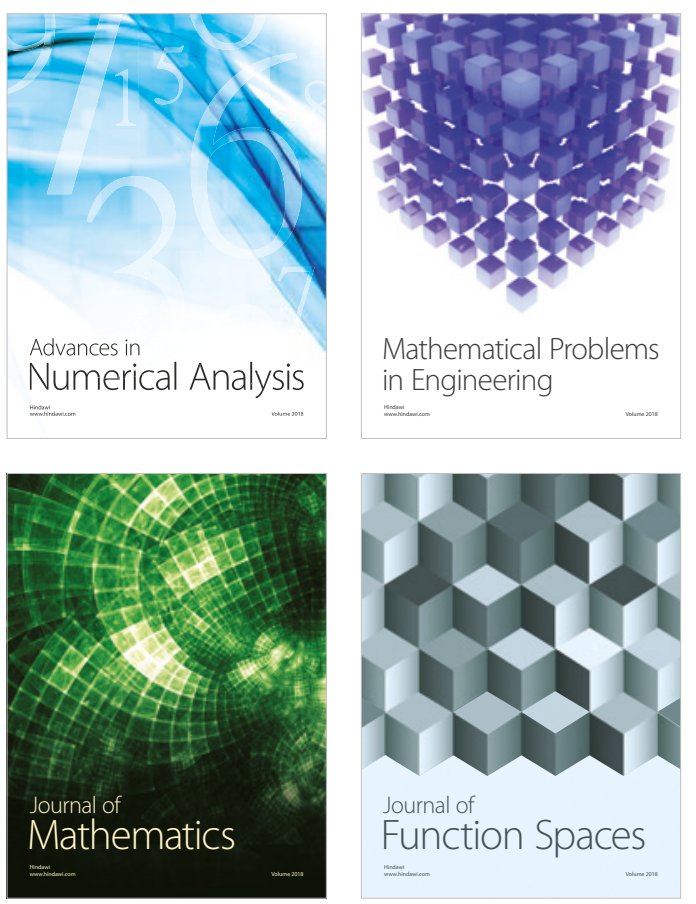

Mathematical Problems in Engineering

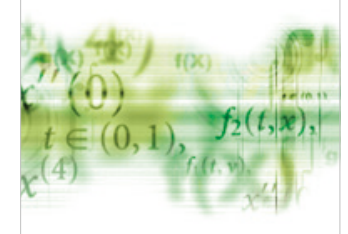

International Journal of

Differential Equations

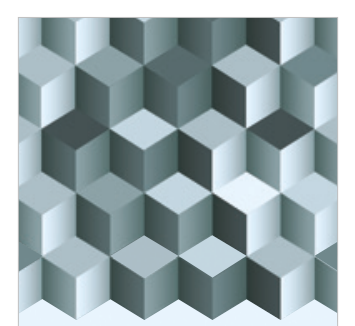

Journal of

Function Spaces

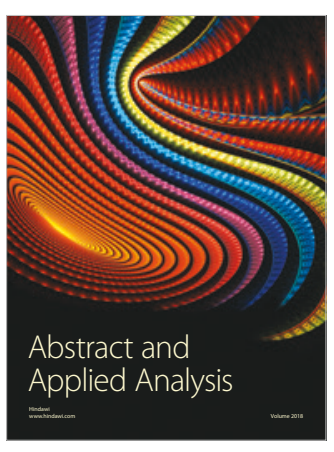

The Scientific

World Journal

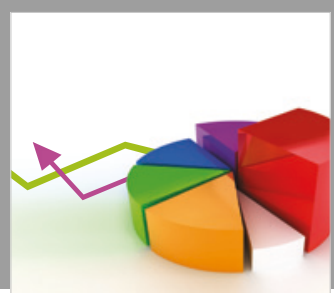

Journal of

Probability and Statistics
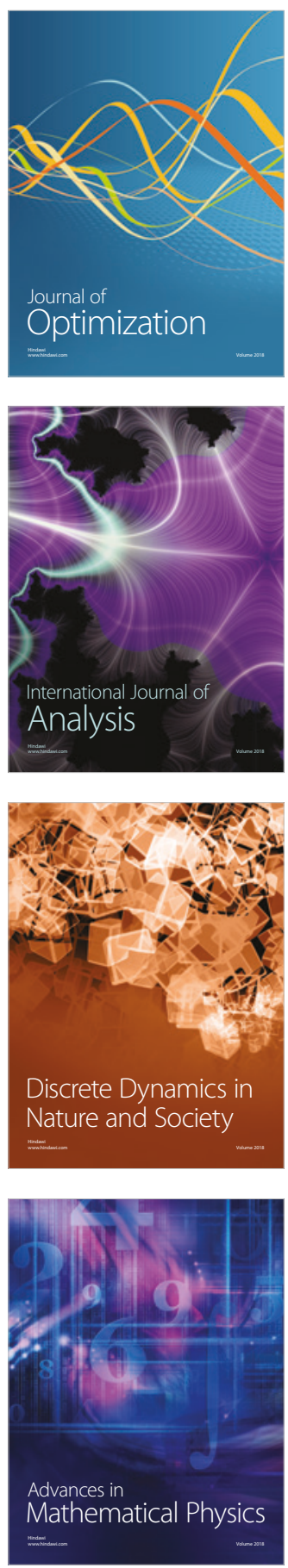